\title{
Late-onset Warfarin Induced Skin Necrosis: In a Hemodialysis Patient
}

Shafiee MA*, Akbarian F, Memon KK, Aarabi M, Shahideh Y, Johnson D and Sharif U

Division of General Internal Medicine, Department of Medicine, Royal College Mentor, Toronto General Hospital, University of Toronto, Toronto, Canada

*Corresponding author: Mohammad A Shafiee, MD, MSc, Assistant Professor, Division of General Internal Medicine, Department of Medicine, Royal College Mentor, Toronto General Hospital, University of Toronto, 200 Elizabeth Street, 14 EN-208, ON, M5G 2C4, Toronto, Canada, Tel: +416-340-4800; E-mail: mohammad.shafiee@uhn.ca

Received date: June 3, 2017; Accepted date: June 19, 2017; Published date: June 20, 2017

Copyright: ( 2017 Shafiee MA, et al. This is an open-access article distributed under the terms of the Creative Commons Attribution License, which permits unrestricted use, distribution, and reproduction in any medium, provided the original author and source are credited.

\begin{abstract}
Chronic dialysis patients frequently present with necrotic skin lesions. The differential diagnoses are broad and complex. The main focus of this paper is differentiating features of two diagnostic entities: Warfarin induced skin necrosis and calciphylaxis as early recognition and treatment may prevent the significant mortality. We discuss a case involving a hemodialysis patient, who developed necrotic skin lesions over her thighs and gluteal areas shortly after restarting warfarin for atrial fibrillation without heparin bridging therapy. Incisional biopsies and pathology report were consistent with warfarin induced skin necrosis. Significant improvement of the lesions following discontinuation of Warfarin was also confirming the diagnosis of Warfarin Induced Skin Necrosis.
\end{abstract}

Keywords: Anticoagulant; Hypercoagulable; Skin necrosis; Case Report

Thrombosis; Calciphylaxis

\section{Abbreviation}

CKD: Chronic Kidney Disease; AF: Atrial Fibrillation; WISN: Warfarin-induced Skin Necrosis; PTH: Parathyroid Hormone; INR: International Normalization Ratio; PCC: Prothrombin Complex Concentrate; CUA: Calcific Uremic Arteriolopathy; NOAC: New Oral Anticoagulant

\section{Introduction}

Warfarin as the first oral anticoagulant has been the mainstay of treatment as well as prevention of thromboembolic disease all over the world and continues to be the most widely used anticoagulation drug even after the introduction of new oral anticoagulants (NOACs) in recent years [1]. NOACs are not appropriate for patients with liver disease or renal insufficiency. Warfarin is still favorable because of its low cost, specific antidote, dose adjustment based on laboratory monitoring, and well-known side effects [2]. Warfarin induced skin necrosis (WISN), is a rare and severe hematologic complication which carries a high risk of morbidity and mortality. It is estimated to occur in $0.01-0.10 \%$ of patients taking oral anticoagulants [3]. Although WISN typically occurs within the first ten days of therapy initiation with a peak incidence between days 3 and 6 , some patients develop the complication months or even years later [4]. Sometimes, the diagnosis of WISN could be challenging, especially in the patients undergoing dialysis therapy for chronic kidney disease. Necrotic skin lesions are common in long-term dialysis patients. This gives rise to a broad and complex differential diagnosis $[5,6]$. One such example is calciphylaxis, and although a different mechanism causes it, shares a similar initial clinical presentation to WISN [7]. We will present a case of a 76-yearold lady with a history of hemodialysis and long-term anticoagulation therapy, which developed necrotic skin lesions after a brief interruption in warfarin therapy.
A 76-year-old lady with the history of multiple co-morbidities including chronic kidney disease (CKD), coronary artery disease (CAD) and atrial fibrillation (AF) presented to emergency with acute ischemic right foot.

She was admitted to hospital for the management of acute exacerbation of chronic kidney disease before her angioplasty. On admission, the patient was alert and oriented. She was on warfarin approximately for the past 11 years for AF, which was stopped before the procedure and was resumed afterward. The patient did not receive heparin at the time of re-initiation of warfarin. Over the next few days, the patient developed several skin lesions over the thighs and gluteal areas bilaterally which initially presented as large, bullous and hemorrhagic areas (Figure 1). A few hours later, primary lesions progressed to necrotic plaques (Figures 2 and 3).

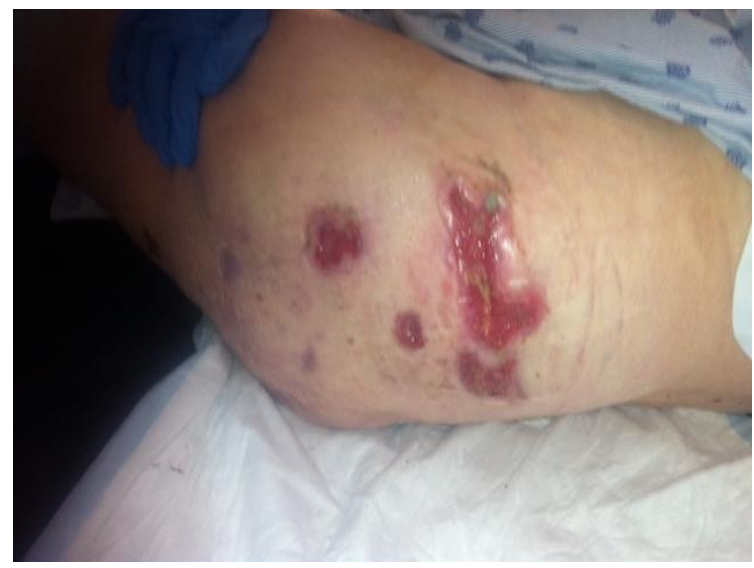

Figure 1: Initial lesions with extensive necrosis over the thighs and buttocks bilaterally. 
Citation: Shafiee MA, Akbarian F, Memon KK, Aarabi M, Shahideh Y, et al. (2017) Late-onset Warfarin Induced Skin Necrosis: In a Hemodialysis

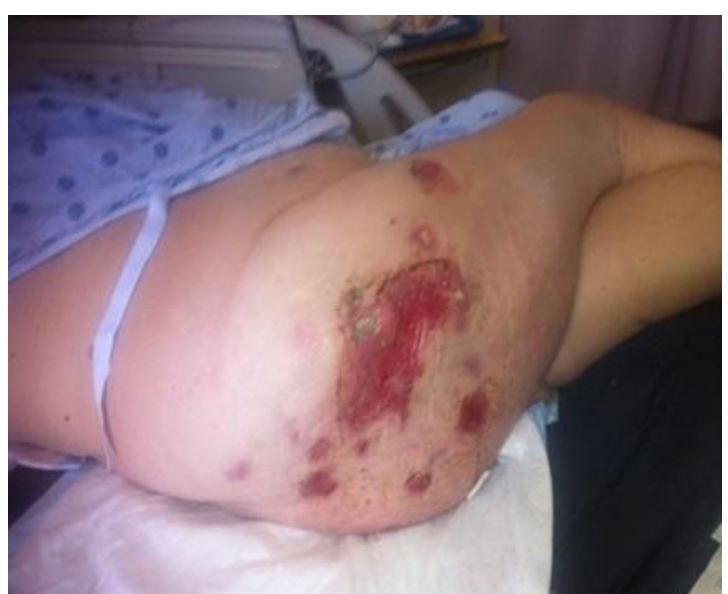

Figure 2: Post debridement with granulation occurring.

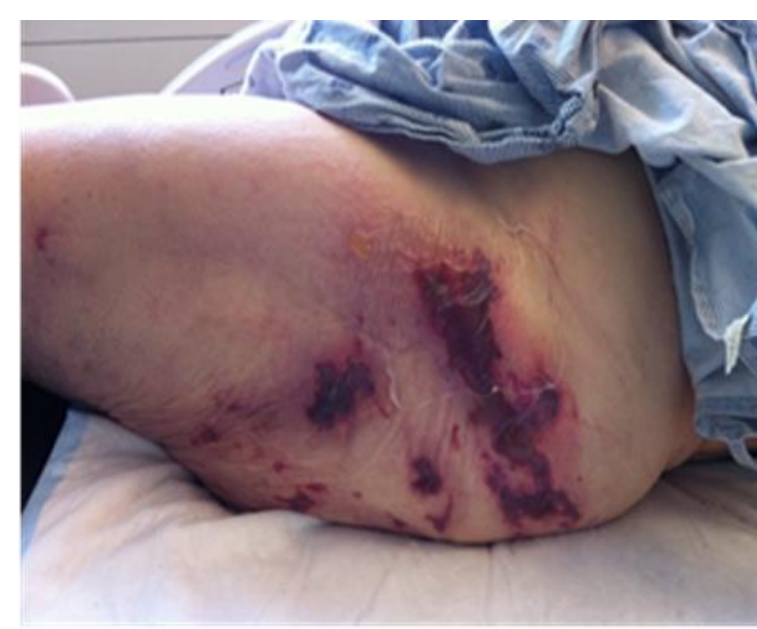

Figure 3: Healing lesion.

Calciphylaxis was considered the initial diagnosis because of elevated parathyroid hormone (PTH) levels and also with regards to her concurrent CKD and hemodialysis therapy. Later, an incisional skin biopsy was performed, and the pathology showed extensive cutaneous necrosis with associated neutrophilic inflammation, without smooth muscle calcification consistent with the clinical impression of WISN (Figures 4 and 5). Also, improvement of lesions while holding warfarin and timing and evolution of lesions after restarting warfarin were further clues for diagnosing warfarin-induced skin necrosis (WISN).

\section{Discussion}

The first case of WISN was described by Flood et al. when the diagnosis of thrombophlebitis migrans disseminate was considered for a necrotic breast tissue [3]. It is now known that the cause of necrotic tissue was a Coumarin derivative called bis-hydroxy coumarin.

WISN usually presents between 3-6 days after initiating warfarin treatment [8]. There are reports of late-onset warfarin-induced skin necrosis occurring after several months or even several years (the longest is 15 years) [3].

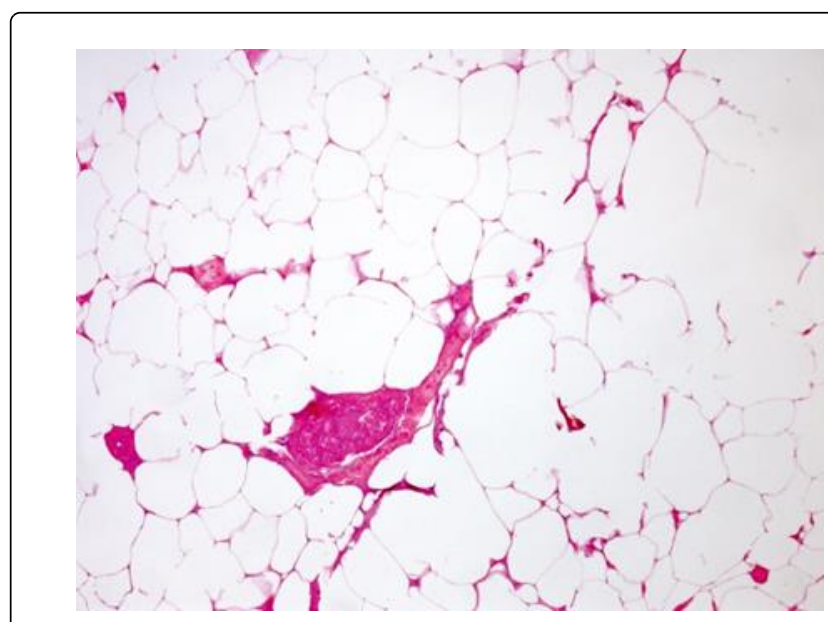

Figure 4: Cutaneous necrosis with vein thrombosis.

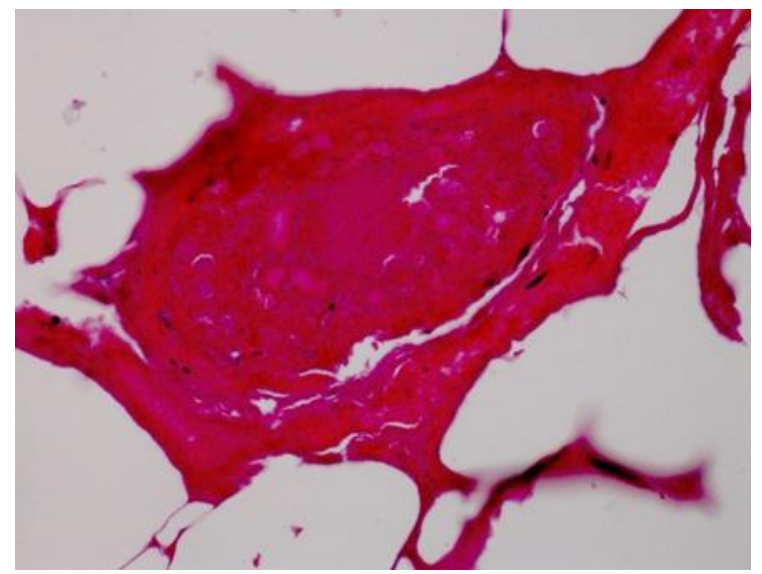

Figure 5: Cutaneous necrosis with vein thrombosis.

The pathogenesis of WISN is not clear; however, the proposed mechanism describes a rapid decline in vitamin K-dependent coagulation factors after warfarin administration. These factors have short half-lives creating an imbalance of procoagulant and anticoagulant proteins [9]. With rapid decrease in the activity level of anticoagulant protein $\mathrm{C}$ (half-life $\sim 8 \mathrm{hrs}$ ) compared to the clotting factors II, IX, and X, which have longer half-lives ( 24-72 hrs), hypercoagulable state results [3]. The resultant hypercoagulable state leads to thrombosis and blood clots in the dermal capillaries which cause skin necrosis due to an interruption in the blood supply to the skin [10]. Although some cases of WISN have been associated with hereditary protein $\mathrm{C}$ deficiency, it has also occurred in other thrombophilic conditions like protein S deficiency, antithrombin III deficiency, and factor V Leiden mutation [11,12]. Occasional cases of late appearance in the course of therapy may be a result of intermittent non-compliance or reintroduction of warfarin, liver dysfunction and drug interactions $[4,11]$.

WISN typically occurs in middle-aged, perimenopausal, obese females being treated for thromboembolic events [10]. Women are 
Page 3 of 4

affected more frequently than men (4:1) for which the reason for this predilection is unclear [13]. The necrotic lesions usually appear in a distinct pattern, with a predilection for fatty tissues such as the breasts, buttocks, thighs, and abdomen [14,15]. Lesions associated with WISN are usually painful, localized and abrupt in onset. Initially, the patient complains of paresthesia or discomfort in the affected areas associated with an erythematous flushes caused by capillary dilation. The lesions develop quickly to manifest a peau d' orange appearance with surrounding edema in the skin and subcutaneous tissue. Underlying petechial hemorrhage rapidly coalesces into ecchymosis, which within hours produces a hemorrhagic bulla, indicative of infarction and gangrenous necrosis. The Escher eventually sloughs, revealing a deep necrotic defect into the subcutaneous fat $[3,7,16]$.

The differential diagnoses of WISN are vast. It must be distinguished from other pathologies such as calciphylaxis, antiphospholipid syndrome, vasculitides and other cutaneous lesions like purple toe syndrome, pyoderma gangrenosum, microembolization, leukocytoclastic vasculitis, necrotizing fasciitis and purpura fulminans which may present similarly during the early stages $[3,17]$.

Histopathology of WISN demonstrates occlusive fibrin thrombi within dermal and subcutaneous veins, venules and capillaries, with endothelial cell damage resulting in diffuse tissue necrosis and hemorrhage [18,19]. The lack of perivascular inflammation and arteriolar thrombosis, differentiates WISN from the vasculitic processes $[15,20]$.

Early factor replacement therapy is used to prevent further thrombosis; however, later factor replacement therapy would be more supportive. Warfarin is typically discontinued; however, its cessation does not affect lesion progression [3]. Therapeutic anticoagulation with IV heparin is used to prevent further thrombosis [7]. The action of warfarin on protein $\mathrm{C}$ is reversed by administering vitamin $\mathrm{K}$ or fresh frozen plasma [15]. Other therapies used are protein $C$ concentrates for deficient patients, prostacyclin [3,7] and a new option 4- factor prothrombin complex concentrate (PCC), which contains the coagulation factors and proteins $\mathrm{C}$ and $\mathrm{S}$ [21]. Small lesions may be allowed to heal by secondary intention. 8 Severe cases may require surgical debridement, skin grafting or even amputation $[3,7,9]$. The three-month mortality rate is approximately $15 \%$ [7].

Calciphylaxis also called calcific uremic arteriolopathy (CUA), is a rare and often fatal complication of end-stage renal disease [5,22] where decreased renal production of 1,25-dihydroxycholecalciferol leads to secondary hyperparathyroidism and hyperphosphatemia [5,7]. The reported incidence rate is $1-4 \%$ in chronic hemodialysis patients $[5,22]$. It is characterized by medial wall calcification of small and medium-sized vessels with intimal proliferation, fibrosis, and thrombosis, primarily in the dermis and subcutaneous fat resulting in ischemia and necrosis [22].

Risk factors for calciphylaxis include female gender, Caucasian race, obesity, diabetes mellitus, elevated serum phosphate levels, hypercalcemia, hyperparathyroidism, low serum albumin levels and warfarin therapy $[5,22,23]$.

The most common sites of involvement overlie dense adipose tissue such as the breast, abdomen and thighs [24,25]. Clinically, patients initially develop superficial violaceous skin lesions that are painful, hyperesthetic or pruritic surrounded by pallor or ecchymoses manifesting a livedo reticularis pattern. This pattern refers to the changes in cutaneous blood flow, which produces bluish-purple retinacular hyperpigmentation on the skin [4]. The initial redness may evolve into intense erythema and then frank necrosis with eschar formation. Gangrenous infection may thereafter develop at the site.

The management of CUA includes pain control, wound care, discontinuation of calcium-based binders, normalization of phosphate, and Para thyroidectomy. Other therapies have included sodium thiosulfate and hyperbaric oxygen administration [24]. The prognosis is often poor with a mortality rate of $60-80 \%$, often from sepsis [7].

\section{Conclusion}

Warfarin as a consequence of its low cost, specific antidote and wellknown side effects is likely the most frequently prescribed anticoagulation despite recently introduced NOACs. Warfarin-induced skin necrosis is one of the most severe and rare complications with a high rate of morbidity and mortality. Patients with underlying partial protein $\mathrm{C}$ deficiency could be asymptomatic, but hold a higher risk of skin necrosis following treatment with warfarin. Therefore, adequate bridge therapy and prompt diagnosis based primarily on clinical judgment with the support of histological evidence are crucial for prognosis.

\section{References}

1. Weitz JI, Semchuk W, Turpie AG, Fisher WD, Kong C, et al. (2015) Trends in prescribing oral anticoagulants in Canada, 2008-2014. Clin Ther 37: 2506-2514.

2. Hanley CM, Kowey PR (2015) Are the novel anticoagulants better than warfarin for patients with atrial fibrillation? J Thorac Dis 7: 165-171.

3. Ward CT, Chavalitanonda N (2006). Atypical Warfarin-induced skin necrosis; Pharmacotherapy 26: 1175-1179.

4. Essex DW, Wynn SS, Jin DK (1998) Late-onset warfarin-induced skin necrosis: case report and review of the literature. Am J Hematol 57: 233-237.

5. Shafiee MA, Akbarian F, Memon KK, Aarabi M, Boroumand B (2015) Dermatologic manifestations in end-stage renal disease. Iran J Kidney Dis 9: 339-353.

6. Piccoli GB, Quaglia M, Quaglino P, Burdese M, Bermond F, et al. (2002) Acute digital gangrene in a long-term dialysis patient - A diagnostic challenge. Med Sci Monit 8: CS83-89.

7. Chacon G, Nguyen T, Khan A, Sinha A, Maddirala S (2010) Warfarininduced skin necrosis mimicking calciphylaxis: A case report and review of the literature. J Drugs Dermatol 9: 859-863.

8. Warkentin TE, Sikov WM, Lillicrap DP (1999) Multicentric warfarininduced skin necrosis complicating heparin-induced thrombocytopenia. Am J Hematol 62: 44-48.

9. Tai CY, Ierardi R, Alexander JB (2004) A case of warfarin skin necrosis despite enoxaparin anticoagulation in a patient with protein $S$ deficiency. Ann Vasc Surg 18: 237-242.

10. Kumar M, Abrina VM, Chittimireddy S (2012) Coumadin-induced skin necrosis in a 64 year-old female despite LMWH bridging therapy. Am J Case Rep 13: 157-159.

11. Parsi K, Younger I, Gallo J (2003) Warfarin-induced skin necrosis associated with acquired protein C deficiency. Australas J Dermatol 44: 57-61.

12. Moll S (2004) Warfarin-induced skin necrosis. British J Haematology 126: 628.

13. Bhaijee F, Wainwright H, Meintjes G, Wilkinson RJ, Todd G, et al. (2010) Warfarin-induced skin necrosis in HIV-1-infected patients with tuberculosis and venous. S Afr Med J 100: 372-377.

14. Yang Y, Algazy KM (1999) Warfarin-induced skin necrosis in a patient with a mutation of the prothrombin gene. N Engl J Med 340: 735-735.

15. Mungalsingh CR, Bomford J, Nayagam J, Masiello M, Ekeowa UI, et al. (2012) Warfarin-induced skin necrosis. Clinical Medicine 12: 90-91. 
Citation: Shafiee MA, Akbarian F, Memon KK, Aarabi M, Shahideh Y, et al. (2017) Late-onset Warfarin Induced Skin Necrosis: In a Hemodialysis Patient. J Kidney 3: 141. doi:10.4172/2472-1220.1000141

Page 4 of 4

16. Kaiber FL, Malucelli TO, Edo RB, Schafranski MD, Akamatsu HT, et al. (2010) Heparin-induced thrombocytopenia and warfarin-induced skin necrosis: Case report. An Bras Dermatol 85: 915-918.

17. Ng T, Tillyer ML (2001) Warfarin-induced skin necrosis associated with Factor V Leiden and protein S deficiency. Clin Lab Haematol 23: 261-264.

18. Gailani D, Reese EP (1999) Anticoagulant-induced skin necrosis in a patient with hereditary deficiency of protein S. Am J Hematol 60 231-236.

19. Voore NK, Rahman A, Sanna S, Bahrain H (2012) Purpuric lesion on the elbow. Cleve Clin J Med 79: 253-254.

20. Martin CM, Engstrom PF, Chandor SB (1970) Skin necrosis associated with warfarin sodium. Calif Med 113: 78-80.
21. Pourdeyhimi N, Bullard Z (2014) Warfarin-induced skin necrosis. Hospital Pharmacy 49: 1044-1048.

22. Hayashi M, Takamatsu I, Kanno Y, Yoshida T, Abe T, et al. (2011) A casecontrol study of calciphylaxis in Japanese end-stage renal disease patients. Nephrol Dial Transplant 27: 1580-1584.

23. Fine A, Zacharias J (2002) Calciphylaxis is usually non-ulcerating: Risk factors, outcome and therapy. Kidney Int 61: 2210-2217.

24. Wilmer WA, Magro CM (2008) Calciphylaxis: Emerging concepts in prevention, diagnosis, and treatment. Semin Dial 15: 172-186.

25. Rockx MA, Sood MM (2009) A necrotic skin lesion in a dialysis patient after the initiation of warfarin therapy: A difficult diagnosis. J Thromb Thrombolysis 29: 130-133. 